\title{
Optimal Dimensional Synthesis of Force Feedback Lower Arm Exoskeletons
}

\author{
Ramazan Unal and Volkan Patoglu
}

\begin{abstract}
This paper presents multi-criteria design optimization of parallel mechanism based force feedback exoskeletons for human forearm and wrist. The optimized devices are aimed to be employed as a high fidelity haptic interfaces. Multiple design objectives are discussed and classified for the devices and the optimization problem to study the trade-offs between these criteria is formulated. Dimensional syntheses are performed for optimal global kinematic and dynamic performance, utilizing a Pareto front based framework, for two spherical parallel mechanisms that satisfy the ergonomic necessities of a human forearm and wrist. Two optimized mechanisms are compared and discussed in the light of multiple design criteria. Finally, kinematic structure and dimensions of an optimal exoskeleton are decided.
\end{abstract}

\section{INTRODUCTION}

Wearable force feedback robotic devices, haptic exoskeletons, are becoming increasingly common as they find widespread use in medical and virtual reality (VR) applications. Utilization of such devices for physical therapy promises to increase effectiveness of conventional rehabilitation approaches.

Design of haptic exoskeletons is a challenging task, since in addition to being ergonomic and light weight, such devices are also required to satisfy the demands of any ideal forcefeedback device: ability withstand human applied forces with very high stiffness and capacity to display a full range of impedances down to the minimum value human can perceive.

If not properly designed, the dynamics of an exoskeleton device can significantly deteriorate the transparency of displayed forces during haptic rendering of virtual environments. Even though parasitic effects due to the device dynamics can be actively compensated using feedback paradigms, such approaches require use of force sensors. Active cancelation approaches suffer from the limited bandwidth of the force sensors, undesired sensor dynamics, sensor actuator co-location and high cost of force sensors. Design optimization studies performed on haptic interfaces can shape the device dynamics in a favorable manner; therefore, these studies can have a significant impact on the overall performance of the haptic display independent of the control algorithm employed.

Multiple performance requirements have to be considered simultaneously while performing design optimization of haptic exoskeletons. Since the performance with respect to many of these criteria cannot be improved without deteriorating others, design trade-offs are inevitable. Determination of an

R. Unal and V. Patoglu are with Faculty of Engineering and Natural Sciences, Mechatronics Program, Sabanc1 University, 34956 İstanbul, Turkey. ramazanunal@su.sabanciuniv.edu, vpatogluesabanciuniv.edu. optimal design with respect to many criteria is a difficult problem and should be handled with formal multi-objective optimization methods that assign trade-offs systematically.

There exists several studies in literature in which multiple competing design criteria have been considered for design of robotic devices. The studies that can be categorized under scalarization methods address the multi-criteria optimization problem in an indirect manner, by first transforming it into a (or a series of) single objective (scalar) problem(s). Among these approaches, Hayward et al. define the relationship between multiple criteria and utilize sensitivities of these criteria to conduct a hierarchical optimization study [1]. Multiple objectives are considered sequentially in [2], [3], [4] by searching for parameter sets resulting in near optimal kinematic performance and then selecting the design exhibiting the best dynamic performance from this reduced parameter space. Task-priority [5], probabilistic weighting [6], composite index [7], and tabular methods [8] are among the other scalarization approaches that consider multiple criteria. Scalarization methods possess the inherent disadvantage of their aggregate objective functions requiring preferences or weights to be determined apriori, ie. before the results of the optimization process are actually known [9].

The alternative approach is classified as pareto methods, which incorporate all optimization criteria within the optimization process and address them simultaneously to find a set of non-dominated design in the objective space. Pareto methods allow the designer to make an informed decision by studying a wide range of options, since they contain solutions that are optimum from an overall standpoint; unlike scalarization techniques that may ignore this trade-off viewpoint. In literature Krefft et al. applied a modified genetic algorithm (GA) based Pareto method to design of parallel mechanisms [3], [10]. Similarly, in [11] GA is applied to multi criteria optimization of a 2-DoF parallel robot. Finally, in [12], [13] authors proposed a multi-objective design framework for optimization of parallel mechanisms based on Normal Boundary Intersection (NBI) method [14]. In [15] the proposed framework is applied to design of a spherical wrist type robot for dual purpose application. The proposed framework is computational efficient, applicable to any set of performance indices, and extendable to include any number of design criteria that is required by the application.

In this paper, selection of kinematic structure and dimensional synthesis of lower arm exoskeletons are conducted using the multi-criteria design optimization framework introduced in [12], [13]. The optimized devices are aimed to be employed as a high fidelity haptic interfaces for human 
forearm and wrist. Multiple design objectives for the devices are discussed and classified for the application scenario at hand, and an optimization problem to study the trade-offs between these criteria is formulated. Dimensional syntheses are performed for optimal global kinematic and dynamic performance of two spherical parallel mechanisms that satisfy the ergonomic necessities of a human forearm and wrist. Then, two mechanisms are compared with respect to the design criteria and advantages/disadvantages of each design are discussed. Finally, kinematic structure and dimensions of an optimal exoskeleton are decided in the light of Paretofront curves generated.

The paper is organized as follows: Section II introduces the kinematic model of human lower arm and discusses the selection two spherical parallel mechanisms as the candidate kinematic structures. Several design objectives are identified and categorized in Section III. Section IV formulates the multi-criteria optimization problems and summarizes the optimization methods used to address them. Section V presents and discusses the results of optimization problems. Finally, Section VI concludes the paper.

\section{KINEMATICS OF HUMAN LOWER ARM AND SPHERICAL PARALLEL MECHANISMS}

The movement of human wrist is quite complex since it is capable of lateral flexion and extensions motions around the radiocarpal and midcarpal joints axes as well as abduction and adduction motions about an axis that passes through the capitate. Moreover the whole human wrist is capable of supination and pronation movements about the axis of the forearm. Even though the rotation axes of these motions are subject to small variations as the joints move, simplified kinematics of the human elbow and wrist can be quite faithfully modeled as a three degrees of freedom (DoF) kinematic chain that allows supination/pronation of the forearm and flexion/extension and abduction/adduction of the wrist joint. In the simplified kinematic model, the axes of rotation for these three motions coincide at a single point on the wrist. Workspace and torque limits of human forearm and wrist are listed in many references including [16].

A kinematic chain that is suitable to serve as an exoskeleton should have rotation axes of its joints coincident with the rotation axes of human wrist when the device is worn by an operator. Moreover, the choice of closed kinematic chains (parallel mechanisms) are preferable over their serial counterparts in satisfying requirements of force feedback applications, since parallel mechanisms possess inherent advantages. Specifically, parallel mechanisms offer compact designs with high stiffness and have low effective inertia since their actuators can be grounded, or placed on parts of the mechanism that experience low accelerations, in many cases. In terms of dynamic performance, high position and force bandwidths are achievable with parallel mechanisms thanks to their light but stiff structure. Besides, parallel mechanisms do not superimpose position errors at joints, hence can achieve high precision.
In order to span an acceptable portion of the natural human wrist and forearm workspace and to ensure alignment of the axes of rotation of human joints with the controlled DoF of the device such that decoupled actuation and measurement of human joint rotations are possible, two closed kinematic chain based mechanisms, namely 3RPSRand 3UPS-S mechanisms, are selected as the candidate kinematic structures of the exoskeleton. Both mechanisms both belong to the larger family of spherical parallel mechanisms (SPMs). Even though there has been important recent advances in the type synthesis of SPMs [17], [18], [19], design and analysis of many of even the most basic types of these mechanisms are still open research topics [20]. 3RPS and 3UPS-S mechanisms are among the few SPMs, whose kinematic and singularity analyses are fully addressed in the literature. Moreover, being compact and allowing for human arm motions without collisions with the device, these two mechanisms are the most suitable SPMs to serve as wearable force feedback devices.

3RㅁS- $\underline{R}$ and 3UPS-S mechanisms are depicted in Figure 1. The 3RPS- $\underline{R}$ mechanism is of hybrid kinematic structure and comprises of a 3 R $\underline{\text { S }}$ parallel wrist in series with an actuated revolute $(\underline{R})$ joint at the base platform of the wrist, while the 3UPS-S mechanism is purely parallel and comprises of a 3UPS parallel wrist coupled with the kinematics of the idealized human wrist that acts as a spherical (S) joint at the moving platform of the mechanism.

The 3RPS platform, first introduced by Lee et al. [21], and further analyzed in [22], consists of five bodies: a base platform $F$, three extensible links $R, S, T$, and a moving platform $W$. The end-effector held by the operator is rigidly attached to the moving platform $W$. Extensible links are connected to the base platform via revolute joints whose axes of rotation are oriented along the tangents of $F$, while the moving platform is connected to the extensible links by means of spherical joints. In this paper, the analysis is limited to a symmetric 3RPS mechanism where the revolute joints and the spherical joints are spaced at $120^{\circ}$ along the circumference of the base platform of radius $R$ and the moving platform of radius $r$, respectively.

The 3R표- $\underline{R}$ mechanism has four DoF corresponding to the height $z$ and Euler angles $\psi_{1}, \psi_{2}$ and $\psi_{3}$ of the moving platform $W$ with respect to the Newtonian reference frame $N$. The rotation of the base platform and the lengths of the extensible links are actuated to control these DoF. The platform possesses limited translational movement transverse to the vertical axis through the base and no singularities for limited values of revolute joint angles $\theta_{i} \in(0, \pi / 2)$ [21]. The 3RPS- $\underline{\mathrm{R}}$ mechanism is first utilized as an exoskeleton device by Gupta et al. [23] and adapted as a rehabilitation device in [16].

The 3UPS-S mechanism, first analyzed in [24], also consists of five bodies: a base platform $N$, three extensible links $R, S, T$, and a moving platform $W$. The end-effector held by the operator is rigidly attached to the moving platform $W$. The wrist of the operator is idealized as a spherical joint and the forearm of the operator is fixed to the base 


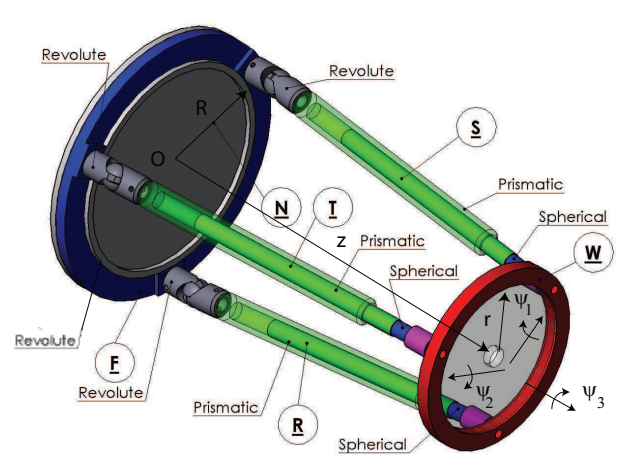

3RPS- $\underline{R}$

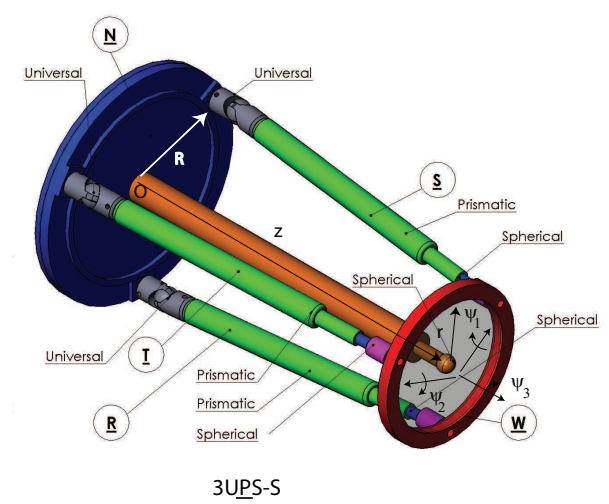

Fig. 1. 3RPS- $\underline{R}$ and 3UPS-S mechanisms in perspective views

platform $N$. Hence, for this mechanism, the human arm counts as a part of the kinematic structure. ${ }^{1}$ Extensible links are connected to the base platform via spherical joints, while the rotating platform is connected to the extensible links (and human forearm) by means of spherical joints (and wrist). In this paper, the analysis is limited to a symmetric 3UPS$\mathrm{S}$ mechanism where the universal joints and the spherical joints are spaced at $120^{\circ}$ along the circumference of the base platform of radius $R$ and the moving platform of radius $r$, respectively. Initial configuration of the 3UPS-S mechanism selected as $\Psi_{1}=\psi_{2}=0$ and $\phi_{3}=90^{\circ}$ so that the mechanism possesses the best kinematic isotropy at the initial configuration.

The 3UPS-S mechanism has three DoF corresponding to Euler angles $\psi_{1}, \psi_{2}$ and $\psi_{3}$ of the moving platform $W$ with respect to the Newtonian reference frame $N$. The lengths of the extensible links are actuated to control these DoF. The moving platform is a distance $z$ from the base platform and does not possess translational movement transverse to the vertical axis through the base. No singularities exist for this mechanism for limited values of revolute joint angles $\theta_{i} \in(0, \pi / 2)$ [20]. The 3UPS-S mechanism is first utilized as an exoskeleton device by Yang et al. [25]

Since the performance of parallel mechanisms is highly sensitive to their dimensions, optimization studies are absolutely necessary for design of these types of mechanisms [26]. Moreover, comparison of two kinematic chains can only be performed once they are both optimized for the same set of performance criteria. In this paper, optimal dimensions for both of these mechanisms will be calculated with respect to multiple design criteria to be detailed in the next section, and then a comparison will be made between the two mechanisms.

\section{DESIGN OBJECTIVES}

Following the terminology of Merlet [26], one can categorize the performance requirements of a mechanism into four distinct groups: Imperative requirements that must be satisfied for any design solution, optimal requirements for

\footnotetext{
${ }^{1}$ Note that kinematics of human arm is not required to be considered in the analysis of 3RPS- $\underline{R}$; since, unlike the case for 3UPS-S, kinematics of human arm only imposes redundant constraints to $3 \underline{R} \underline{P}-\underline{R}$.
}

which a maximal value of the index is required, primary requirements which take place in the specifications but can be modified to some extend to ensure a design solution, and secondary requirements which do not appear in the specifications but can be utilized to choose between multiple design solutions.

Ensuring the safety and complying with the ergonomic needs of the human operator are two imperative design requirements every exoskeleton device must satisfy. Safety is typically assured by recruitment of back-drivable impedance type devices with force/torque limits implemented in software, while the ergonomy of the device is considered at the kinematic synthesis level. Predetermined workspace volumes are imposed for the application and being capable of spanning all of the desired workspace and ensuring alignment of the axes of rotation of human joints with the controlled DoF of the device, both 3R $\underline{P} S-\underline{R}$ and 3UPS-S mechanisms satisfy the ergonomic requirements of a lower arm exoskeleton. The absence of singularities in the workspace is another imperative design requirement the both mechanisms satisfy, that ensures the forward and inverse kinematics of the robots can be solved uniquely at each point within the workspace.

The performance requirements to be optimized are highly dependent on the final use of the device. For a high fidelity haptic interface, kinematic/dynamic isotropy and stiffness of the device (including the actuator and transmission compliance) should be maximized while effective moving mass should be minimized to achieve high force bandwidths and a uniform "feel" for the device.

Optimal performance of mechanisms are quantified through study of several design matrices, including kinematic Jacobian $(J)$ and mass matrix $(M)$. In this paper global performance measures, characterizing the performance of a manipulator over the entire workspace are considered.

To quantify the kinematic/dynamic performance of the haptic interface global isotropy index $(G I I)$ and global dynamic index $(G D I)$ are proposed in [4]. Both of these indices are conservative workspace inclusive worst-case performance measures that are intolerant of poor performance over the entire workspace. An optimal GII results in a uniform kinematic Jacobian matrix, while optimizing $G D I$ minimizes the effective mass matrix of the system. Other commonly 
used global isotropy indices include average $(A I I)$ and standard deviation $(S D I I)$ of the local isotropy index over the workspace. Since the stiffness of both system are dominated by the compliance of the transmission and actuators, optimization for a Jacobian matrix with high $(A I I)$ will result in maximization of the stiffness of the device.

Since entries of kinematic Jacobian and mass matrices of the $3 R \underline{R}-\underline{R}$ mechanism are not homogenous in units, scaling factors need to be introduced for this mechanism. Scaling factors eliminate the physical units and normalize the elements of these matrices as fractions of their maximum values so that comparable relative values are ensured [4].

The primary requirement for the wearable exoskeleton is selected as the workspace volume index [26], the ratio between the workspace volume and the volume of the robot. Even though predetermined workspace volume is imposed as an imperative requirement, a large workspace volume index is desirable to reduce the collisions of the device with the operator and the environment. The weight of the device is highly dependent on the selection of the actuators, more so than the link lengths; hence, there exists some flexibility on deciding the total mass of the kinematic structure.

Finally, the secondary requirements for both devices include low backlash, low-friction, high back-drivability, and low manufacturing costs. Friction, backlash and backdrivability are mainly influenced by the selection of the actuators and the transmission, while choice of link lengths may have an influence on manufacturing costs.

\section{OPTIMIZATION}

\section{A. Multi-criteria Optimization Problem}

For optimal dimensioning of the exoskeleton as a high fidelity haptic interface, two objective functions characterizing the kinematic and dynamic performance of the mechanism are considered. The objective of optimization is to maximize the worst kinematic isotropy of the mechanism (GII) while simultaneously minimizing the effective mass ( $\max$ singular value of the effective mass matrix or $G D I$ ).

The negative null form of the multi-objective optimization problem can be stated as

$$
\begin{gathered}
\max \mathbf{F}(\boldsymbol{\alpha}, \boldsymbol{\beta}, \boldsymbol{\gamma}) \\
\mathbf{G}(\boldsymbol{\alpha}, \boldsymbol{\beta}) \leq 0 \\
\boldsymbol{\alpha}_{l}<\boldsymbol{\alpha}<\boldsymbol{\alpha}_{u}
\end{gathered}
$$

where $\mathbf{F}$ represents the column matrix of objective functions that depend on the design variables $\boldsymbol{\alpha}$, parameters $\boldsymbol{\beta}$, and workspace positions $\gamma$. Symbol $\mathbf{G}$ represents the inequality constraint function that also depend on design variables and parameters. Finally, $\boldsymbol{\alpha}_{l}$ and $\boldsymbol{\alpha}_{u}$ correspond to the lower and upper bounds of the design variables, respectively.

The symmetric 3R $\underline{R S}-\underline{R}$ and 3UPS-S mechanisms both have two parameters $\beta_{1}=r$ and $\beta_{2}=W$, where $r$ is the radius of the moving platform and selected according to statistical data on human joint sizes listed in [16] and $W=\psi_{1} \times \psi_{2} \times \psi_{3}$ represents the predetermined workspace. The workspace is assumed symmetric and set as $W=30^{\circ} \times$ $30^{\circ} \times 60^{\circ}$ for the design of the high fidelity haptic interface.
The optimization problem has two design variables: the ratio of the moving and base platform radii $\alpha_{1}=r / R$ and the perpendicular distance of the moving platform $\alpha_{2}=z$. Upper $\boldsymbol{\alpha}_{\boldsymbol{u}}$ and lower $\boldsymbol{\alpha}_{\boldsymbol{l}}$ limits on the design parameters are imposed according to statistical data on human arm [16].

The column matrix of objective functions for the haptic interface $\mathbf{F}$ is given as

$$
\mathbf{F}=\left[\begin{array}{ll}
G I I & G D I
\end{array}\right]^{T}
$$

while the constraints are imposed during kinematic analysis to ensure the closed kinematic chain for the 3RPS and 3UPS$S$ platforms and the positive perpendicular travel pose of the mechanisms.

\section{B. Solution Methods}

The multi-criteria design optimizations of the $3 R \underline{P S}-\underline{R}$ and 3UPS-S parallel mechanisms are conducted using the framework introduced in [12], [13]. This optimization framework for parallel mechanisms is based on NBI method [14] to efficiently obtain the Pareto-front hyper-surfaces characterizing the design trade-offs. Based on gradient techniques, the approach is more efficient than other methods to obtain a well represented Pareto-front hyper-surface including aggregate methods such as weighted sums and evolutionary optimization approaches such as GAs.

The proposed framework is computational efficient, as the NBI approach attacks the geometric problem directly by solving for single-objective constrained subproblems to obtain uniformly distributed points on the hyper-surface. The number of subproblems can be adjusted by defining resolution of the grid that maps to the number of points on the Pareto-front hyper-surface. As the number of points increases, the computational time increases linearly, but since the method assumes spatial coherence and uses solution of a subproblem to initialize the next subproblem, convergence time for each subproblem may decrease resulting in further computational efficiency. The method does not suffer from clumping of solution in the objective space and results in exceptionally uniform distributed points on the Paretofront hyper-surface without requiring any tuning of the core algorithm.

The approach is generalizable to other sets of performance indices, and trivially extends to handle any number of objective functions. Moreover, the framework can solve for points on the non-convex regions of Pareto-front hyper-surfaces, a feature that is missing from the weighted sum methods.

Limitations of the technique exist due to reliance of the NBI on equality constraints. It is possible for NBI not to find a solution on the true Pareto-front hyper-surface, converging to a local optima. In such a case, post processing on the solutions of NBI subproblems can be employed to filter out undesired dominated solutions. Moreover, NBI assumes sufficient smoothness of the geometric problem at hand so that gradient techniques can be employed. However, it has also been demonstrated in the literature that the method performs remarkably well even for non-smooth geometries of the objective space [27]. 


\section{RESULTS AND DISCUSSION}

Figure 2(a) and (b) present the distribution of GII and $G D I$ of $3 R \underline{P}$ S- $\underline{R}$ device over the parameter space. Similarly, Figure 2(c) and (d) present the distribution of GII and $G D I$ of 3 UPS-S device over the parameter space. From these plots one can observe that both $G I I$ and $G D I$ indices vary an important amount for different parameter values of the 3RPS-R mechanism, while the variance is much less for the 3UPS-S mechanism.

In fact, dynamic isotropy (GDI) of the 3UPS-S device is almost uniform over the parameter space and effects of parameter changes being minimal. The situation is quite different for the $3 R \underline{R}-\underline{R}$ mechanism and such behaviour is expected since the 3RPS platform part of this device rotates around the fixed base, causing the change in link lengths have an important effect on the effective inertia. In Figure 2(d) the variation of GDI for the 3UPS-S device is negligible, while such variation is about 10 times higher for the $3 R \underline{R} \underline{P}-\underline{R}$ device in Figure 2(b).

Kinematic isotropy $(G I I)$ of the 3 RPSS-R mechanism also varies substantially as the parameters are changed. Moreover, the 3RPS- $\underline{R}$ device can achieve much larger GII values than the 3UPS-S device. The variation of kinematic isotropy of the 3 UPS-S mechanism under different parameter values is important and the characteristic of this change is complex.

To characterize the trade-off between the single objective solution of each mechanism, Pareto-front curves for the biobjective optimization problem is constructed in Figure 3 employing the NBI method. Figure 3(a) presents uniformly distributed points on the Pareto-front curve characterizing the trade-off between GII and GDI for the 3R $\underline{\mathrm{P} S}-\underline{\mathrm{R}}$ device, while Figure 3(b) depicts the same plot for the 3UPS-S device. Since the GDI values changes only a negligible amount for the 3UPS-S, the Pareto-front curve for this device is effectively a straight line along which only the parameter $\rho$ changes from 0.36 to 0.47 almost monotonically while the perpendicular platform distance $z=100 \mathrm{~mm}$. The Paretofront of the $3 \mathrm{R} \underline{\mathrm{PS}}-\underline{\mathrm{R}}$ is a convex curve along which values of optimal parameters vary from $\rho=0.56, z=100 \mathrm{~mm}$ to $\rho=0.36, z=200 \mathrm{~mm}$ in a non-trivial manner.

From these plots one can conclude that 3UPS-S mechanism possesses a much better dynamic performance than

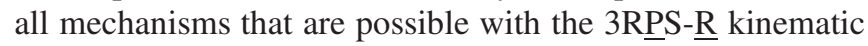
structure. Further analysis of the maximum singular values of $M$ for both mechanisms reveals that effective inertias of both mechanisms are quite similar, assuming identical links are used during their construction. On the other hand, best worst case kinematic performance $(G I I)$ of the $3 \underline{R} \underline{P S}-\underline{R}$ mechanisms are about two times better than kinematic performances of all possible 3UPS-S devices. Hence, the 3RPS$\underline{\mathrm{R}}$ kinematic structure is preferred for the exoskeleton device. Placing and equal amount of emphasis on both kinematic and dynamic performance criteria, an optimal design is selected with parameter values $\rho=0.56, z=146 \mathrm{~mm}$ that is marked on Figure 3 with a star.

\section{CONCLUSIONS}

Optimal dimensional synthesis of spherical parallel mechanism based lower arm exoskeletons for forearm and wrist motions, to be employed as a high fidelity haptic interfaces, are conducted. After identifying the relevant design criteria for the application at hand, an optimization problem to study the trade-offs between these criteria is formulated. A multi-criteria design optimization framework for parallel mechanisms is applied to efficiently obtain the Pareto-front hyper-surfaces between conflicting criteria. Two kinematic structures are compared and considering the primary and secondary design criteria, an "optimal" design is selected utilizing the Pareto-front curves generated.

\section{ACKNOWLEDGMENTS}

The authors gratefully acknowledge the support by TÜBİTAK with grant number 107 M337.

\section{REFERENCES}

[1] V. Hayward, J. Choksi, G. Lanvin, and C. Ramstein, "Design and multi-objective optimization of a linkage for a haptic interface," in Advances in Robot Kinematics, 1994, pp. 352-359.

[2] G. Alici and B. Shirinzadeh, "Optimum synthesis of planar parallel manipulators based on kinematic isotropy and force balancing," Robotica, vol. 22, no. 1, pp. 97-108, 2004.

[3] M. Krefft, H. Kerle, and J. Hesselbach, "The assesment of parallel mechanisms - it is not only kinematics," Production Engineering, vol. 12, no. 1, pp. 173-173, 2005.

[4] L. Stocco, S. E. Salcudean, and F. Sassani, "Fast constrained global minimax optimization of robot parameters," Robotica, vol. 16, no. 6, pp. 595-605, 1998.

[5] W. Chen, Q. Zhang, Z. Zhao, and W. A. Gruver, "Optimizing multiple performance criteria in redundant manipulators by subtask-priority control," in IEEE International Conference on Systems, Man and Cybernetics, 1995, pp. 2534-2539.

[6] S. McGhee, T. F. Chan, R. V. Dubey, and R. L. Kress, "Probabilitybased weighting of performance criteria for a redundant manipulator," in IEEE International Conference on Robotics and Automation, 1994, pp. 1887-1894.

[7] J. H. Lee, K. S. Eom, B.-J. Yi, and I. H. Suh, "Design of a new 6-DoF parallel haptic device," in IEEE International Conference on Robotics and Automation, vol. 1, 2001, pp. 886-891.

[8] J. Yoon and J. Ryu, "Design, fabrication, and evaluation of a new haptic device using a parallel mechanism," IEEE Transactions on Mechatronics, vol. 6, no. 3, pp. 221-233, 2001.

[9] O. L. de Weck, "Multiobjective optimization: History and promise," in China-Japan-Korea Joint Symposium on Optimization of Structural and Mechanical Systems, ser. Invited Keynote Paper, 2004.

[10] M. Krefft and J. Hesselbach, "Elastodynamic optimization of parallel kinematics," in IEEE International Conference on Automation Science and Engineering, 2005, pp. 357-362.

[11] S.-D. Stan, V. Maties, and R. Balan, "Optimal design of 2 DoF parallel kinematics machines," in Applied Mathematics and Mechanics, 2006, pp. 705-706.

[12] R. Unal, G. Kiziltas, and V. Patoglu, "A multi-criteria design optimization framework for haptic interfaces," in IEEE International Symposium on Haptic Interfaces for Virtual Environments and Teleoperator Systems, 2008.

[13] - "Multi-criteria design optimization of parallel robots," in IEEE International Conference on Cybernetics and Intelligent Systems and IEEE International Conference on Robotics, Automation and Mechatronics, CIS-RAM 2008, 2008, p. to appear.

[14] I. Das and J. E. Dennis, "Normal-boundary intersection: A new method for generating the pareto surface in nonlinear multi-criteria optimization problems," SIAM Journal on Optimization, vol. 8, no. 3, pp. 631-65, 1996.

[15] R. Unal and V. Patoglu, "Optimal dimensional synthesis of a dual purpose haptic interface," in Lecture Notes in Computer Science, Springer, 2008, p. to appear. 


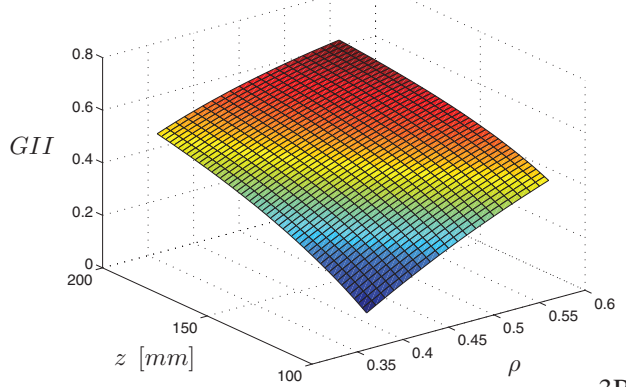

(a)

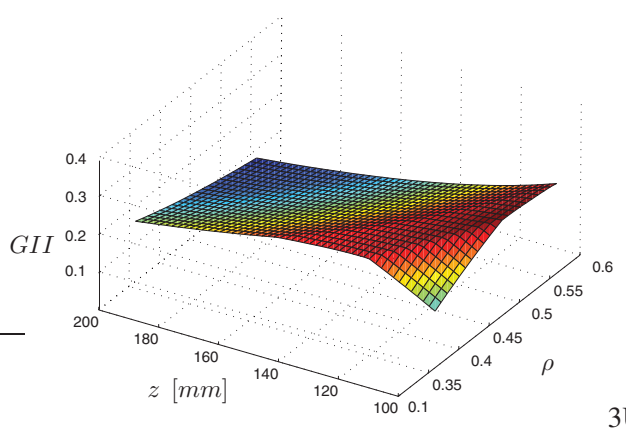

(c)

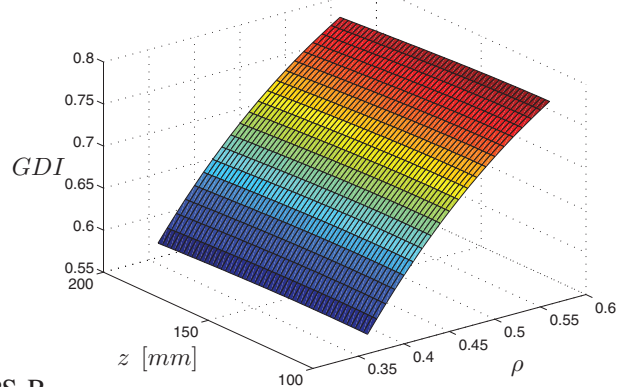

(b)

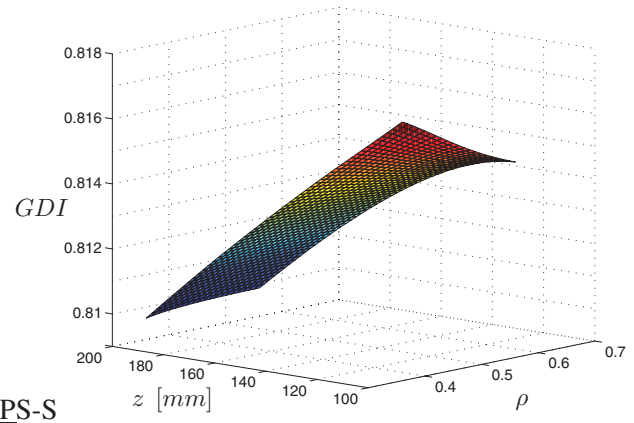

(d)

Fig. 2. Change of $G I I$ and $G D I$ for $3 \underline{R} \underline{P S}-\underline{R}$ and $3 U \underline{P} S-S$ mechanisms over parameter space

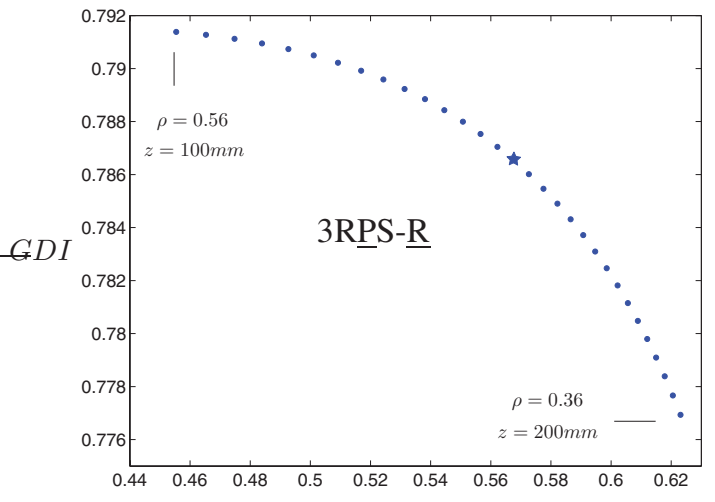

(a)

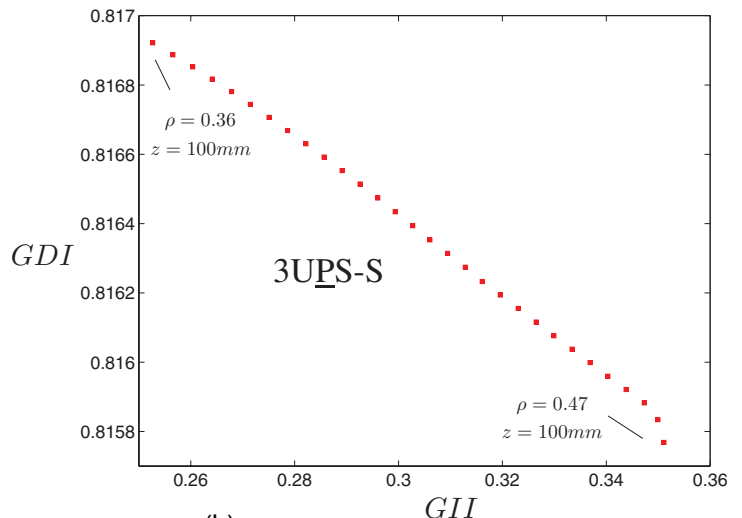

(b)

Fig. 3. Pareto-front curves for 3R표 $\underline{R}$ and 3UPS-S mechanisms

[16] A. Gupta, V. Patoglu, M. K. O’Malley, and C. M. Burgar, "Design, control and performance of RiceWrist: A force feedback wrist exoskeleton for rehabilitation and training," International Journal of Robotics Research, Special Issue on Machines for Human Assistance and Augmentation, to appear.

[17] X. Kong and C. M. Gosselin, "Type synthesis of 3-DoF spherical parallel manipulators based on screw theory," in ASME Design Engineering Technical Conferences, 2002.

[18] M. Karouia and J. M. Herve, "A family of novel orinetational 3DoF parallel robots," in CISM-IFToMM Symposium on Robot Design, Dynamics, and Control, 2002, pp. 359-368.

[19] R. Di Gregorio, "Kinematics of a new spherical parallel manipulator with three equal legs: The 3-URC wrist," Journal of Robotic Systems, vol. 18, no. 5, pp. 213-219, 2001.

[20] I. A. Bonev and C. M. Gosselin, "Singularity loci of spherical parallel mechanisms," in IEEE International Conference on Robotics and Automation, 2005, pp. 2957-2962.

[21] K. M. Lee and D. K. Shah, "Kinematic analysis of a three degreesof-freedom in-parallel actuated manipulator," IEEE Transactions on
Robotics and Automation, vol. 4, no. 3, pp. 354-360, 1988.

[22] C. H. Liu and S. Cheng, "Direct singular positions of 3RPS parallel manipulators," ASME Journal of Mechanical Design, no. 126, pp. 1006-1016, 2004.

[23] A. Gupta and M. K. O'Malley, "Design of a haptic arm exoskeleton for training and rehabilitation," IEEE Transactions on Mechatronics, vol. 11, no. 3, 2006.

[24] C. Innocenti and V. Parenti-Castelli, "Echelon form solution of direct kinematics for the general fully parallel spherical wrist," Mechanisms and Machine Theory, vol. 28, no. 4, pp. 553-561, 1993.

[25] G. Yang, H. L. Ho, W. Chen, W. Lin, S. H. Yeo, and M. S. Kurbanhusen, "A haptic device wearable on a human arm," in IEEE Conference on Robotics, Automation and Mechatronics, 2004, pp. 243-247.

[26] J. Merlet, Parallel Robots, 2nd ed. Springer, 2006.

[27] E. Rigoni and S. Poles, "NBI and MOGA-II, two complementary algorithms for multi-objective optimizations," in Practical Approaches to Multi-Objective Optimization, 2005. 